\title{
Denis Jeffrey (dir.). (2015). Laïcité et signes religieux à l'école. Presses de I'Université Laval
}

doi:10.18162/fp.2015.a70

\section{RECENSION}

Louvrage dirigé par Denis Jeffrey s'inscrit dans le débat sur la laïcité et le port du signe religieux qui a fait tant de vagues notamment avec la Charte des valeurs du Parti Québécois. Celle-ci prenait la position controversée d'interdire le port des signes religieux dans la fonction publique. Ce livre propose donc une réflexion autour de ces délicates questions de la laïcité et des signes religieux dans un espace séculier et scolaire.

Dans une première partie de l'ouvrage, les auteurs se montrent critiques envers la vision de la laïcité proposée par les politiciens voulant interdire le port des signes religieux. Selon Micheline Milot, l'interdiction du signe religieux contredit l'esprit de la laïcité qui prônait à son origine : "l'égalité entre tous les membres de la société $[\ldots] »($ p. 12). L'exigence de neutralité des fonctionnaires induirait même une discrimination envers les groupes religieux "visibles ». Jean Baubérot rappelle que la visibilité dérangeante du religieux n'est pas nouvelle et, en France, le discours autour du voile musulman aujourd'hui ressemble à celui tenu un siècle auparavant sur la soutane du prêtre (acte de prosélytisme, signe de soumission). Toutefois, si la soutane n'a pas été interdite, le voile l'est, notamment dans les écoles. Selon l'auteur, l'école, étant « le lieu du catéchisme républicain enseigné à la jeunesse », peut expliquer cela (p. 27). Georges Leroux remet en question des arguments prônant la prohibition du signe religieux. Il utilise, entre autres, l'exemple du hijab à l'école que les enseignants ne devraient pas porter du fait de leur grande influence sur 
les élèves, selon certains. Pour Leroux, au contraire, «[...] le port du signe religieux signale qu'un a priori serait possible et invite à y être attentif, ce qui n'est pas le cas pour des enseignants qui ne portent pas de tels signes » (p. 38). Jocelyn Maclure, lui aussi, déconstruit des arguments des tenants de la Charte des valeurs. Il leur reproche, entre autres, une méconnaissance de la religion et de la liberté de conscience. Si pour les prohibitionnistes l'interdiction du signe religieux durant les heures de travail est un "sacrifice raisonnable ", ce dernier est loin dêtre mineur dans des religions où le signe religieux est fondamental. Gérard Bouchard, bien que partisan d'une laïcité inclusive, ouvre la porte à l'interdiction dans certains cas. Il évoque des motifs supérieurs pouvant la justifier, dont « la nécessité de protéger au maximum la crédibilité de certaines institutions fondamentales». Un peu plus loin, Pierre-Luc St-Onge explique que le débat qui entoure la laïcité est devenu un corollaire à celui sur l'identité des Québécois francophones. La première est alors reprise et instrumentalisée par certains partis politiques. Mais cette laïcité politisée est souvent unidimensionnelle et l'ouverture aux minorités y est mise à mal, constate l'auteur. Enfin, Guy Ménard conclut cette partie sur la laïcité en nous présentant deux matrices qui ont tiraillé le débat au Québec. La première, de source britannique, prône les droits individuels et la seconde, plus ancrée dans la tradition française, se base sur des principes tels la liberté. Guy Ménard presse le Québec d'« inventer une sorte de voie originale entre les deux grandes matrices $[\ldots] »$.

Dans la deuxième partie, les auteurs concentrent leur réflexion autour du voile musulman au cœur des débats. Ainsi, Denis Jeffrey s'interroge sur le lien entre éthique des fonctionnaires et port d'un signe religieux tel que le voile. Il est fallacieux, selon lui, de penser qu'un fonctionnaire soit impartial seulement par le port du voile. Il démontre que le port du signe religieux n'est pas garant de l'éthique professionnelle du fonctionnaire. Meryem Sellami, quant à elle, explique qu'en France, le voile islamique "glisse d'une morale sexuelle et puritaine à un principe fondateur de l'identité musulmane » au début $\mathrm{du} \mathrm{XX}^{\mathrm{e}}$ siècle. Et cela n'est pas sans incidence pour les jeunes musulmanes tiraillées, par une crise identitaire personnelle, entre une culture d'origine où l'honneur familial est important et une culture occidentale où la femme dispose de son corps. Jean-René Milot et Raymonde Venditti poursuivent dans le même sens et nous proposent de poser un regard sur la multiplicité des sens derrière le voile. Chaque femme qui porte le voile islamique est une personne unique et son parcours de vie donne sens à son choix. De son côté, David Koussens montre les conséquences politiques et sociales d'une loi française de 2010 interdisant « la dissimulation du visage dans l'espace public » (p. 94). Cette loi, contestée devant les tribunaux européens, a été validée. Ce faisant, la Cour européenne laisse aux États le choix de l'acceptation ou non du port de voile (intégral ici) au nom du vivre-ensemble de chaque société. " [E]lle légitime - même de façon ponctuelle - une forme de contrôle social des minorités ", ce qui est inquiétant (p.102).

La troisième partie se penche sur la position éthique des enseignants face à ce signe religieux. Le premier article collectif examine quelques arguments en faveur d'une prohibition du port du signe religieux pour les enseignants, comme le principe de neutralité de l'État, et ils se demandent s'ils la justifient en soi. En règle générale, leur réponse est plutôt négative, mais les auteurs ouvrent la porte à l'interdiction dans certains cas au nom de la confiance du public envers les institutions. Selon eux, par exemple, dans l'enseignement religieux des écoles publiques, l'enseignant devrait s'abstenir d'afficher ses convictions personnelles. Dans les articles suivants, c'est d'ailleurs l'enseignant d'ECR (éthique et culture religieuse) qui est au centre des attentions. Si Marjorie Paradis pense que «les enseignants pourront 
aborder en toute impartialité les éléments de contenu du programme, quelle que soit leur appartenance religieuse, soit-elle visible ou non [...] » (p. 142), d'autres auteurs, comme Nancy Bouchard et Mireille Estivalèzes, pensent que l'enseignant d'ECR devrait plutôt s'abstenir. Bouchard s'interroge néanmoins sur la question de l'abstention de dévoiler, pour l'enseignant, ses propres convictions. La réponse, loin d'être claire, oscille entre la question de l'impartialité de l'enseignant et celle de son authenticité. Elle conclut toutefois que la prudence devrait diriger les enseignants vers l'abstention. Estivalèzes est plus ferme : le devoir de réserve demandé aux enseignants d'ECR rend impossible le port de signe religieux. Selon elle, la liberté intellectuelle pour discuter des grandes questions éthiques « sera favorisée par l'absence de signes religieux chez les enseignants » (p. 161).

Louis LeVasseur s'intéresse au port du signe religieux, mais par les élèves cette fois. Il oriente son questionnement autour de la relation entre construction identitaire, rapport au savoir et rapport à l'institution. Il réfléchit sur la façon dont l'école négocie avec ces délicates questions. Au cœur de cette négociation, il y a le phénomène de la socialisation qui, par les institutions, est «producteur à la fois de contraintes et de libertés » (p. 167).

Sivane Hirsch, partant de sa propre expérience de «juive laique », montre comment la société occidentale peine à comprendre les religiosités, comme le voile par exemple, tant elle est déconnectée de sa propre religion et de l'expérience spirituelle. Elle déplore également un manque d'ouverture à une laïcité plurielle où il serait possible d'être, par exemple, juive laïque et non pas l'un ou l'autre.

Finalement, dans une conclusion très intéressante, Denis Jeffrey reprend les principaux arguments prohibitionnistes, par exemple l'égalité entre les sexes ou l'intégration sociale. À ces arguments, il donne une réponse antiprohibitionniste.

Il est assez difficile de rendre justice à un tel ouvrage collectif en si peu d'espace. La richesse et la densité de la réflexion des auteurs ne peuvent se résumer à quelques lignes. Disons, pour conclure, que ce livre est un indispensable pour nourrir et comprendre le débat sur la laïcité et le port du signe religieux. Si les auteurs sont majoritairement antiprohibitionnistes, leurs arguments sont de poids. Quelques auteurs ouvrent néanmoins la porte à une réserve, notamment de la part des enseignants, et leur position apporte, elle aussi, son lot de questions et de remises en question.

\section{Pour citer cet article}

Harvengt, D. (2015). Jeffrey, D. (dir.) (2014). Laïcité et signes religieux à l'école. Presses de l'Université Laval. Formation et profession, 23(3), 233-235. http://dx.doi.org/10.18162/fp.2015.a70 